\title{
Méthode de construction de bases spectrales généralisées pour l'approximation de problèmes stochastiques
}

\author{
ANTHONY NOUY ${ }^{\mathrm{a}}$ \\ Institut de recherche en Génie civil et Mécanique (GeM), Université de Nantes Atlantique, École Centrale de Nantes, \\ UMR CNRS 6183, 2 rue de la Houssinière, BP 92208, 44322 Nantes Cedex 3, France
}

Reçu le 15 mars 2007, accepté le 7 juin 2007

\begin{abstract}
Résumé - Les méthodes éléments-finis stochastiques de type Galerkin conduisent généralement à la résolution de problèmes de très grande taille. Les coûts de calcul et de stockage mémoire engendrés limitent encore leur utilisation à une faible dimension stochastique. Nous proposons ici une méthode d'approximation pour la résolution d'équations aux dérivées partielles stochastiques qui tente de répondre à ces difficultés. Cette méthode peut être interprétée comme une technique de décomposition spectrale généralisée. Elle consiste à décomposer la solution sur une base réduite dont on peut prouver l'optimalité vis-à-vis de l'opérateur et du second membre du problème stochastique. Un algorithme adapté est proposé pour la construction de cette décomposition. Il nécessite uniquement la résolution d'un très faible nombre de problèmes déterministes et d'équations stochastiques.
\end{abstract}

Mots clés : Mécanique numérique probabiliste / éléments-finis stochastiques / décomposition spectrale généralisée / Karhunen-Loeve / bases réduites

\begin{abstract}
Construction of generalized spectral bases for the approximate resolution of stochastic problems. Galerkin stochastic finite elements methods, when dealing with high stochastic dimension, lead to the resolution of huge systems of equations. Induced calculation costs and memory requirements still limit their use to a low stochastic dimension. Here, we propose a method which tries to overcome these difficulties. This method can be interpreted as a generalized spectral decomposition technique. It consists in decomposing the solution on a reduced basis, which can be proved to be optimal with respect to the operator and right-hand side of the stochastic problem. An ad hoc algorithm is proposed for the construction of the decomposition. It only needs for the resolution of a very small set of uncoupled deterministic problems and stochastic equations.
\end{abstract}

Key words: Computational stochastic mechanics / stochastic finite-elements / generalized spectral decomposition / Karhunen-Loeve / reduced basis

\section{Introduction}

Les méthodes éléments-finis stochastiques offrent aujourd'hui un outil robuste pour la résolution de problèmes stochastiques. Les approches de type Galerkin au niveau stochastique [1-3] permettent d'obtenir une solution d'une grande qualité. La recherche de cette solution conduit cependant à résoudre des systèmes de très grande taille. Les coûts de calcul (en temps et en stockage mémoire) engendrés par les techniques de résolution classiques de type Krylov [4-6] limitent l'utilisation de ces approches à une faible dimension au niveau stochastique.

\footnotetext{
a Auteur correspondant : anthony.nouy@univ-nantes.fr
}

Une méthode alternative pour la résolution de problèmes éléments-finis stochastiques a récemment été proposée [7]. Elle est inspirée d'une méthode de résolution d'équations d'évolution [8]. La solution y est décomposée sous la forme d'une somme de fonctions, chaque fonction étant le produit d'une fonction déterministe et d'une fonction aléatoire scalaire. Contrairement aux approches classiques, ni les fonctions déterministes ni les fonctions aléatoires ne sont fixées a priori. Elles sont définies de telle manière à minimiser le nombre de fonctions nécessaires dans la décomposition pour une précision donnée. On montre alors que les fonctions sont solutions d'un problème qui peut être interprété comme un problème aux valeurs propres « étendu ». L'approximation obtenue peut ainsi être interprétée comme une décomposition spectrale 
généralisée de la solution. Cette méthode généralise en quelque sorte la méthode de Karhunen-Loève à la résolution d'un problème stochastique. Elle permet une réduction importante des coûts de calcul par rapport aux approches classiques. De plus, elle conduit naturellement à la construction d'une base réduite de fonctions déterministes (ou de fonctions stochastiques) qui peut être judicieusement exploitée dans le cadre de multirésolutions.

Dans la section 2, nous rappelons la formulation et l'approximation classique d'un problème stochastique. Dans la section 3, nous introduisons les bases de la méthode de décomposition spectrale généralisée ainsi qu'un algorithme de construction adapté. Nous évoquons dans la section 4 l'intérêt d'une réutilisation des bases spectrales dans le cadre de problèmes non-linéaires. Enfin, nous illustrons la méthode dans la section 5 en l'appliquant à un problème de thermique non-linéaire.

\section{Formulation et approximation d'un problème stochastique}

\subsection{Formulation d'un problème stochastique}

La modélisation probabiliste d'un problème de physique se ramène généralement à identifier les sources d'aléas en fonction d'un nombre fini de variables aléatoires. Cela conduit à définir un espace probabilisé de dimension finie adapté à l'étude, noté $(\Theta, \mathcal{B}, P)$, où $\Theta$ désigne l'espace des événements élémentaires, $\mathcal{B}$ une tribu d'événements et $P$ une mesure de probabilité. On note $\theta \in \Theta$ un événement élémentaire. On peut alors classiquement introduire une formulation faible au niveau déterministe d'un problème de physique sous la forme : trouver une variable aléatoire $u(\theta)$ à valeur dans un espace fonctionnel $\mathcal{V}$ tel que l'on ait presque sûrement :

$$
a(u(\theta), v ; \theta)=b(v ; \theta) \quad \forall v \in \mathcal{V}
$$

où $a$ est une forme semilinéaire et $b$ une forme linéaire sur $\mathcal{V}$, ces formes dépendant de l'événement élémentaire $\theta$. On suppose ici que $\mathcal{V}$ est un espace fonctionnel indépendant de l'événement élémentaire. On notera que dans le cas où $u(\theta)$ est un champ défini sur un domaine spatial aléatoire, cette hypothèse nécessite une reformulation du problème [9]. Ayant introduit un espace $\mathcal{S}$ de variables aléatoires ad hoc, la formulation faible du problème peut s'écrire (cf. e.g. [10]) : trouver $u \in \mathcal{V} \otimes \mathcal{S}$ tel que

$$
A(u, v)=B(v) \quad \forall v \in \mathcal{V} \otimes \mathcal{S}
$$

où

$$
\begin{aligned}
& A(u, v)=\int_{\Theta} a(u(\theta), v(\theta) ; \theta) \mathrm{d} P(\theta):=E(a(u, v)), \\
& B(v)=\int_{\Theta} b(v(\theta) ; \theta) \mathrm{d} P(\theta):=E(b(v)) .
\end{aligned}
$$

$E(\cdot)$ désigne l'espérance mathématique. On considère pour l'instant que $A$ est une forme bilinéaire. Le cas nonlinéaire sera introduit dans la section 4 .

\subsection{Approximation de Galerkin et méthodes de résolution}

La semi-discrétisation déterministe du problème (1) consiste à définir un espace d'approximation de dimension finie $\mathcal{V}_{n} \subset \mathcal{V}$. On note $\left\{\varphi_{i}\right\}_{i=1}^{n}$ une base de cet espace. L'approximation sera donc recherchée sous la forme $u=\sum_{i} u_{i}(\theta) \varphi_{i}=\boldsymbol{\varphi} \boldsymbol{u}(\theta)$, où $\boldsymbol{u}(\theta)=\left(u_{1}(\theta) \ldots u_{n}(\theta)\right)^{T}$. Dans le cas de problèmes linéaires, l'approximation de Galerkin est alors définie par le problème suivant : trouver $\boldsymbol{u}(\theta) \in \mathbb{R}^{n} \otimes \mathcal{S}$ tel que

$$
E\left(\boldsymbol{v}^{T} \boldsymbol{A} \boldsymbol{u}\right)=E\left(\boldsymbol{v}^{T} \boldsymbol{b}\right) \quad \forall \boldsymbol{v} \in \mathbb{R}^{n} \otimes \mathcal{S}
$$

où $\boldsymbol{b}: \Theta \rightarrow \mathbb{R}^{n}$ est un vecteur aléatoire et où $\boldsymbol{A}: \Theta \rightarrow$ $\mathbb{R}^{n \times n}$ est une matrice aléatoire dont les composantes sont définies par :

$$
(\boldsymbol{b}(\theta))_{i}=b\left(\varphi_{i} ; \theta\right) \quad \text { et } \quad(\boldsymbol{A}(\theta))_{i j}=a\left(\varphi_{j}, \varphi_{i} ; \theta\right)
$$

$\mathrm{Au}$ niveau stochastique, on définit un espace d'approximation $\mathcal{S}_{P}=\left\{v=\sum_{\alpha \in \mathcal{I}_{P}} v_{\alpha} H_{\alpha}(\theta), v_{\alpha} \in \mathbb{R}, H_{\alpha} \in\right.$ $\mathcal{S}$ \} de dimension finie $P$. On utilise classiquement une approximation par chaos polynômial $[1,11]$, où la base d'approximation $\left\{H_{\alpha}\right\}$ est constituée de polynômes orthogonaux pour le produit scalaire naturel sur $\mathcal{S}$, ou encore une approximation polynômiale par morceaux $[2,12]$. L'approximation de Galerkin $\boldsymbol{u} \in \mathbb{R}^{n} \otimes \mathcal{S}_{P}$ est alors déterminée par la résolution du système d'équations linéaires suivant :

$$
\sum_{\alpha \in \mathcal{I}_{P}} E\left(\boldsymbol{A} H_{\alpha} H_{\beta}\right) \boldsymbol{u}_{\alpha}=E\left(H_{\beta} \boldsymbol{b}\right) \quad \forall \beta \in \mathcal{I}_{P}
$$

Le système (5) est un système de très grande taille $n \times P$. Les techniques de résolution classiquement utilisées sont des techniques itératives de type Krylov (gradient conjugué préconditionné (PCG), conjugate gradient square (CGS), ...) [4-6]. Ces algorithmes profitent de la structure creuse du système. Cependant, les coûts de résolution engendrés limitent l'utilisation de ces techniques à une faible dimension au niveau stochastique. De plus, le coût de stockage mémoire de la solution (et éventuellement des sous espaces de Krylov) devient considérable dans le cas de problèmes de grande dimension.

La technique proposée par la suite permet de pallier ces inconvénients tout en conservant la robustesse et la précision des approches de Galerkin au niveau stochastique.

\section{Méthode de décomposition spectrale généralisée}

\subsection{Principe}

L'idée de la méthode de décomposition spectrale généralisée (GSD) consiste à chercher une approximation du problème (4) sous la forme

$$
\boldsymbol{u}(\theta) \approx \sum_{i=1}^{M} \lambda_{i}(\theta) \boldsymbol{U}_{i}
$$


où les $\lambda_{i} \in \mathcal{S}_{P}$ sont des variables aléatoires et les $\boldsymbol{U}_{i} \in \mathbb{R}^{n}$ des vecteurs déterministes. Une décomposition de ce type sera dite optimale si le nombre de termes $M$ est minimum pour une qualité donnée de l'approximation. L'ensemble des vecteurs déterministes (resp. des variables aléatoires) peut alors être considéré comme une base réduite optimale déterministe (resp. stochastique). Un premier intérêt de cette décomposition apparaît immédiatement : le coût de stockage mémoire d'une solution est divisé par un facteur $\frac{n \times P}{M(n+P)}$. Si $M$ reste faible, le gain devient donc considérable quand les dimensions $n$ et $P$ augmentent.

Deux questions clés se posent alors naturellement : comment définir la décomposition « optimale » et comment la calculer? En fait, la décomposition obtenue dépend de ce qu'on entend par « optimal ». Si la solution était connue, la meilleure approximation serait classiquement définie en minimisant la distance à la solution au sens d'une norme sur $\mathbb{R}^{n} \otimes \mathcal{S}$. Il est bien connu que la meilleure approximation $\boldsymbol{u}^{(M)}$ au sens de la norme naturelle sur $L^{2}\left(\Theta, d P ; \mathbb{R}^{n}\right)$ est la décomposition de KarhunenLoeve (ou décomposition spectrale classique) tronquée à l'ordre $M$ [3]. Bien sûr, la solution $\boldsymbol{u}$ n'étant pas connue a priori, ce type de décomposition n'est pas accessible directement.

Nous introduisons par la suite une manière simple et intuitive de définir a priori cette décomposition ainsi qu'un algorithme de construction adapté. La méthode qui en découle généralise la méthode de décomposition spectrale classique.

\subsection{Définition de la décomposition spectrale généralisée}

En notant $\boldsymbol{W}=\left(\boldsymbol{U}_{1} \ldots \boldsymbol{U}_{M}\right) \in \mathbb{R}^{n \times M}$ et $\boldsymbol{\Lambda}=$ $\left(\lambda_{1} \ldots \lambda_{M}\right)^{T} \in \mathbb{R}^{M} \otimes \mathcal{S}_{P}$, la décomposition recherchée s'écrit $\boldsymbol{u}=\boldsymbol{W} \boldsymbol{\Lambda}$. On choisit alors de définir la « meilleure » approximation de ce type par les deux propriétés d'orthogonalité suivantes :

$$
\begin{aligned}
& E\left(\boldsymbol{\Lambda}^{T}\left(\widetilde{\boldsymbol{W}}^{T} \boldsymbol{A} \boldsymbol{W} \boldsymbol{\Lambda}\right)\right)=E\left(\boldsymbol{\Lambda}^{T} \widetilde{\boldsymbol{W}}^{T} \boldsymbol{b}\right), \forall \widetilde{\boldsymbol{W}} \in \mathbb{R}^{n \times M} \\
& E\left(\widetilde{\boldsymbol{\Lambda}}^{T}\left(\boldsymbol{W}^{T} \boldsymbol{A} \boldsymbol{W} \boldsymbol{\Lambda}\right)\right)=E\left(\widetilde{\boldsymbol{\Lambda}}^{T} \boldsymbol{W}^{T} \boldsymbol{b}\right), \forall \widetilde{\boldsymbol{\Lambda}} \in \mathbb{R}^{M} \otimes \mathcal{S}_{P}
\end{aligned}
$$

Si $\boldsymbol{\Lambda}$ (resp. $\boldsymbol{W}$ ) était fixé, l'équation (7) (resp. (8)) définirait l'approximation de Galerkin naturelle. Ici, aucune des fonctions n'étant fixée a priori, on cherchera naturellement des fonctions vérifiant ces équations simultanément.

Dans le cas de problèmes symétriques, s'écrivant comme un problème de minimisation d'une fonctionnelle, le meilleur choix de fonctions $(\boldsymbol{W}, \boldsymbol{\Lambda})$ est naturellement celui minimisant la fonctionnelle. Dans ce cas, les équations (7) et (8) expriment simplement les conditions de stationnarité de la fonctionnelle. On montre alors que la meilleure décomposition vérifie la propriété suivante (cf. [7]) : $\boldsymbol{W}$ maximise la fonctionnelle $R(\boldsymbol{W})$ définie par

$$
\begin{aligned}
& R(\boldsymbol{W})=\operatorname{Trace}(\boldsymbol{R}(\boldsymbol{W})) \\
& \operatorname{avec} \boldsymbol{R}(\boldsymbol{W})=E\left(\left(\boldsymbol{W}^{T} \boldsymbol{A} \boldsymbol{W}\right)^{-1}\left(\boldsymbol{W}^{T} \boldsymbol{b b}^{T} \boldsymbol{W}\right)\right)
\end{aligned}
$$

et $\boldsymbol{\Lambda}$ est obtenue par l'équation (8). $R(\boldsymbol{W})$ (resp. $R(\boldsymbol{W})$ ) s'interprète comme un quotient de Rayleigh matriciel (resp. scalaire) «étendu ». La définition de la meilleure approximation est donc liée à la résolution d'un problème aux valeurs propres « étendu », la meilleure approximation étant celle associée aux $M$ plus grandes valeurs propres « généralisées ». L'approximation obtenue est dans ce cas la meilleure approximation du type (6) au sens de l'opérateur et du second membre. Pour construire l'approximation (6), il reste à proposer un algorithme capable de capter efficacement le haut de ce « spectre généralisé ».

Remarque. Dans le cas d'un opérateur $\boldsymbol{A}$ symétrique déterministe, on montre que la meilleure décomposition est solution d'un problème aux valeurs propres généralisé classique $\boldsymbol{A} \boldsymbol{W}=E\left(\boldsymbol{b} \boldsymbol{b}^{T}\right) \boldsymbol{W} \boldsymbol{R}(\boldsymbol{W})$ où $\boldsymbol{R}(\boldsymbol{W})$ est le quotient de Rayleigh matriciel classique. La meilleure décomposition spectrale est alors une décomposition spectrale classique du second membre $\boldsymbol{b}$ dans la métrique induite par $\boldsymbol{A}$.

\subsection{Algorithme de construction de la décomposition spectrale généralisée}

L'algorithme 1 proposé est inspiré de la méthode des puissances pour la résolution des problèmes aux valeurs propres classiques. Les couples $\left(\boldsymbol{U}_{i}, \lambda_{i}\right)$ sont ici construits les uns après les autres. Les étapes 3 à 7 correspondent à une résolution itérative du système $((7),(8))$ pour la recherche d'un nouveau couple $\left(\boldsymbol{U}_{i}, \lambda_{i}\right)$. Peu d'itérations suffisent généralement à obtenir une bonne approximation de ce couple. En pratique, on choisit $k_{\max }=3$. L'étape 8, qui correspond à une réactualisation des fonctions stochastiques, est facultative. Cependant, elle permet souvent de réduire le nombre $M$ de couples nécessaires pour une précision donnée.

On notera que l'algorithme proposé nécessite d'une part la résolution de problèmes déterministes (étape 4) et d'autre part de problèmes stochastiques sur une base réduite de fonctions déterministes (étapes 6 et 9). Cela conduit à un coût de calcul considérablement réduit par rapport aux techniques de résolution classiques.

Remarque. Dans le cas d'un opérateur $\boldsymbol{A}$ symétrique déterministe, l'algorithme 1 correspond à une méthode des puissances classique [13] pour la résolution du problème aux valeurs propres. On notera que dans ce cas, l'étape 9 de réactualisation est sans effet. Dans le cas d'un opérateur non-symétrique, l'algorithme 1 n'est pas le plus approprié mais permet cependant de capter relativement efficacement le haut du spectre généralisé (cf. [7]).

Algorithme 1. Power-type Generalized Spectral Decomposition with Updating (PU-GSD).

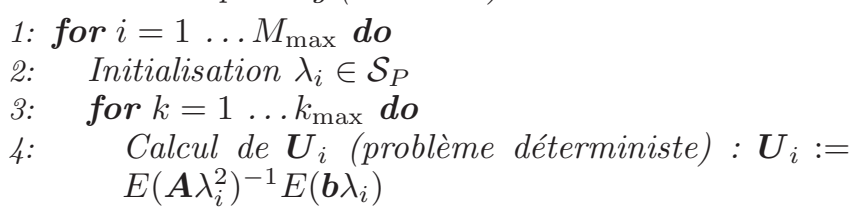


5:

Normalisation de $\boldsymbol{U}_{i}$ et vérification de la convergence

6: Calcul de $\lambda_{i}$ (équation stochastique) : $E\left(\tilde{\lambda}\left(\boldsymbol{U}_{i}^{T} \boldsymbol{A} \boldsymbol{U}_{i}\right) \lambda_{i}\right)=E\left(\tilde{\lambda}\left(\boldsymbol{U}_{i}^{T} \boldsymbol{b}\right)\right), \forall \tilde{\lambda} \in \mathcal{S}_{P}$

\%: $\quad$ end for

8: Actualisation de $\boldsymbol{W}=\left(\boldsymbol{U}_{1} \ldots \boldsymbol{U}_{i}\right)$ et $\boldsymbol{\Lambda}=$ $\left(\lambda_{1} \ldots \lambda_{i}\right)^{T}$

9: Réactualisation de $\boldsymbol{\Lambda}$ en résolvant (8) (système réduit d'équations stochastiques)

10: Réactualisation de $\boldsymbol{b}$ et vérification de la convergence

11: end for

\section{Problèmes non-linéaires : réutilisation des bases réduites spectrales}

Dans le cas de problèmes non-linéaires, la formulation discrétisée du problème (1) peut s'écrire :

$$
E\left(\boldsymbol{v}^{T} \mathcal{A}(\boldsymbol{u})\right)=E\left(\boldsymbol{v}^{T} \boldsymbol{b}\right) \quad \forall \boldsymbol{v} \in \mathbb{R}^{n} \otimes \mathcal{S}_{P}
$$

où $\mathcal{A}$ est un opérateur non-linéaire défini par

$$
E\left(\boldsymbol{v}^{T} \mathcal{A}(\boldsymbol{u})\right)=E(a(\boldsymbol{\varphi} \boldsymbol{u}, \boldsymbol{\varphi} \boldsymbol{v} ; \theta))
$$

Ce système est classiquement résolu par un algorithme itératif. En notant $\boldsymbol{u}^{(k)}$ l'approximation à l'itération $k$, l'itéré suivant $\boldsymbol{u}^{(k+1)} \in \mathbb{R}^{n} \otimes \mathcal{S}_{P}$ est solution du système : $\forall \boldsymbol{v} \in \mathbb{R}^{n} \otimes \mathcal{S}_{P}$

$$
E\left(\boldsymbol{v}^{T} \boldsymbol{A}^{(k)} \boldsymbol{u}^{(k+1)}\right)=E\left(\boldsymbol{v}^{T}\left(\boldsymbol{b}-\mathcal{A}\left(\boldsymbol{u}^{(k)}\right)+\boldsymbol{A}^{(k)} \boldsymbol{u}^{(k)}\right)\right)
$$

où $\boldsymbol{A}^{(k)}$ est une matrice dépendant du solveur non-linéaire utilisé. Dans le cas d'un algorithme de Newton-Raphson, cette matrice est classiquement prise comme la dérivée de Gâteaux de $\mathcal{A}$, définie $\forall \boldsymbol{v}, \boldsymbol{w} \in \mathbb{R}^{n} \otimes \mathcal{S}_{P}$ par :

$$
E\left(\boldsymbol{v}^{T} \boldsymbol{A}^{(k)} \boldsymbol{w}\right)=\lim _{\epsilon \rightarrow 0} \frac{1}{\epsilon} E\left(\boldsymbol{v}^{T}\left(\mathcal{A}\left(\boldsymbol{u}^{(k)}+\epsilon \boldsymbol{w}\right)-\mathcal{A}\left(\boldsymbol{u}^{(k)}\right)\right)\right)
$$

La résolution du problème non-linéaire se ramène donc à la résolution d'un ensemble de problèmes stochastiques linéaires du type (4). L'algorithme 1 peut ainsi être utilisé pour la résolution de chacun de ces systèmes. Un grand intérêt de l'approche présentée est que les bases spectrales généralisées construites au cours des itérations peuvent être stockées et réutilisées pour les résolutions successives. Ces bases s'avèrent très pertinentes vis-à-vis de l'opérateur et du second membre du problème. La réutilisation devient donc très efficace lorsque l'opérateur et le second membre des problèmes linéaires successifs varient peu d'une itération sur l'autre. En pratique, cette réactualisation s'opère en rajoutant au début de l'algorithme 1 une étape d'initialisation. Cette dernière correspond à l'étape 9 où $\boldsymbol{W}$ désigne la matrice dont les vecteurs colonnes constituent la base réduite de vecteurs construits au cours des résolutions linéaires précédentes.

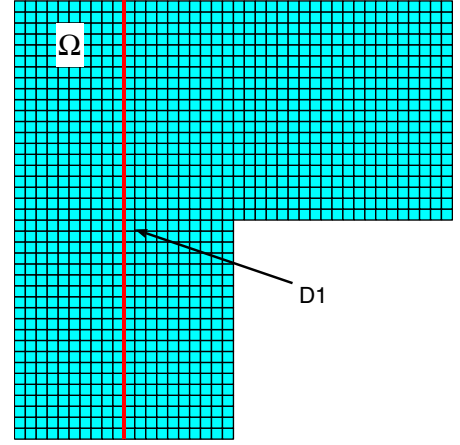

Fig. 1. Présentation du problème et maillage éléments-finis.

\section{Exemple : problème de thermique non-linéaire}

On considère un problème de thermique non-linéaire défini sur le domaine $\Omega$ (Fig. 1). Des conditions homogènes de Dirichlet sont imposées sur la frontière $\partial \Omega$. On utilise au niveau spatial une approximation élémentsfinis $\mathcal{P}_{1}$ (quadrangle à 4 nœuds) avec $n=1121$ degrés de liberté. La température $u(x, \theta)$ semi-discrétisée au niveau déterministe est recherchée sous la forme $u(x, \theta)=$ $\boldsymbol{\varphi}(x) \boldsymbol{u}(\theta), \boldsymbol{u} \in \mathbb{R}^{n} \otimes \mathcal{S}$, où $\boldsymbol{\varphi}$ désigne le vecteur ligne des fonctions d'interpolation éléments-finis. Les formes semilinéaire et linéaire du problème (1) sont définies par

$$
\begin{aligned}
& a(u, v ; \theta)=\int_{\Omega} \nabla v \cdot\left(\kappa_{0}(\theta)+\kappa_{1}(\theta) u^{2}\right) \nabla u \mathrm{~d} x \\
& b(v ; \theta)=\int_{\Omega} f(\theta) v \mathrm{~d} x
\end{aligned}
$$

où $f$ est un terme source de chaleur homogène et $\kappa_{0}$ et $\kappa_{1}$ des paramètres matériau. On modélise $f, \kappa_{0}$ et $\kappa_{1}$ par 3 variables aléatoires indépendantes : $f(\theta)=\mathcal{N}(1 ; 0,2)^{1}$, $\kappa_{0}=U(0,7 ; 1,3)$ et $\kappa_{1}=U(0,7 ; 1,3)^{2}$.

On choisit au niveau stochastique une approximation par chaos polynômial généralisé de degré $p=3$ en dimension $m=3$ [11]. L'espace d'approximation $\mathcal{S}_{P}$ est donc de dimension $P=\frac{(m+p) !}{m ! p !}$.

Le problème est résolu par un algorithme de NewtonRaphson avec une tolérance de $10^{-4}$. Chaque problème linéaire (11) est résolu avec une précision relative de $10^{-5}$. On notera que ces problèmes linéaires sont nonsymétriques. L'algorithme (PU-GSD) proposé est comparé avec un algorithme classique Conjugate Gradient Square (CGS) [13]. Dans le cas où les bases spectrales sont réutilisées, on applique systématiquement une procédure de tri basée sur une décomposition spectrale classique.

L'algorithme (PU-GSD), avec réutilisation et tri des modes déterministes, conduit à convergence à une décomposition spectrale en $M=10$ modes. La figure 2 montre les 6 premiers modes. On notera que ces 6 modes

\footnotetext{
${ }^{1} \mathcal{N}(\mu ; \sigma)$ désigne une variable gaussienne de moyenne $\mu$ et d'écart-type $\sigma$.

${ }^{2} U(\alpha ; \beta)$ désigne une variable uniforme sur $[\alpha, \beta]$.
} 

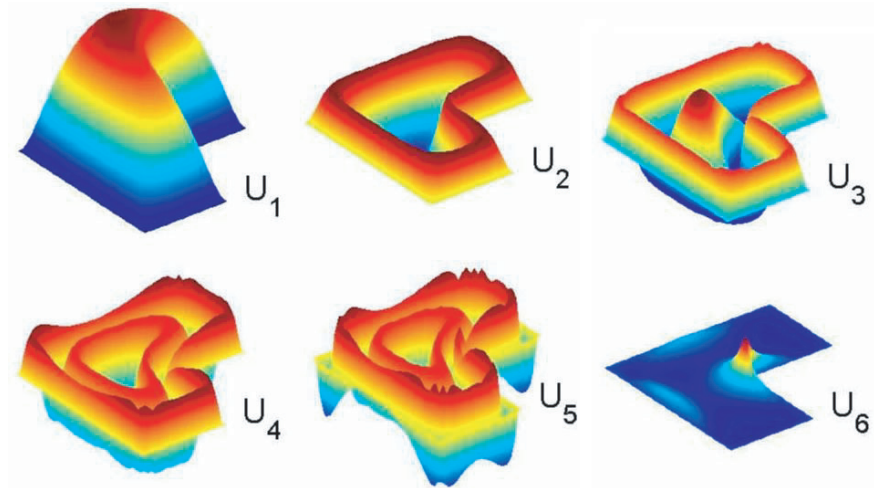

Fig. 2. Modes $\left\{\boldsymbol{U}_{i}\right\}_{i=1}^{6}$ obtenues par PU-GSD avec réutilisation et tri des bases réduites déterministes.

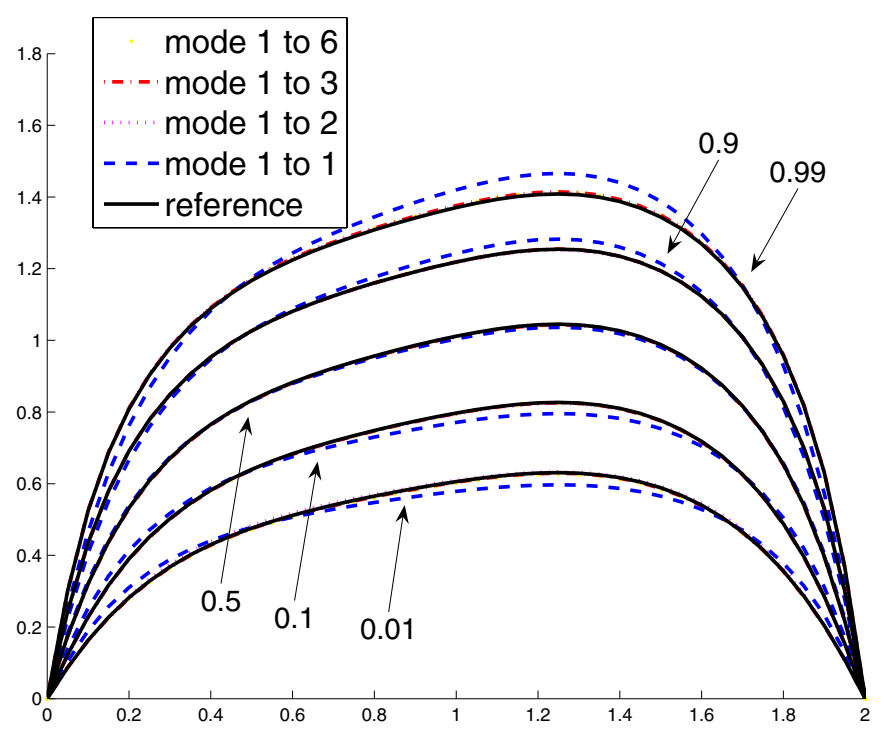

Fig. 3. Quantiles $[0,01,0,1,0,5,0,9,0,99]$ sur la droite $D_{1}$ en fonction du nombre de modes $M$ de la décomposition.

sont les plus significatifs puisque la solution spectrale tronquée à $M=6$ conduit à une erreur de $10^{-4}$. La figure 3 montre les quantiles de la solution le long de la droite $D_{1}$ (Fig. 1) en fonction du nombre de modes $M$ retenus dans la décomposition. On observe que les deux premiers modes $(M=2)$ permettent déjà d'obtenir une très bonne précision sur les quantiles 0,01 et 0,99 .

La figure 4 montre le nombre de nouveaux couples $\left(\boldsymbol{U}_{i}, \lambda_{i}\right)$ construits à chaque itération de NewtonRaphson. On remarque que pour la résolution du premier système linéaire, un seul couple suffit à représenter la solution exacte, ce qui était prévisible. On remarque également qu'avec la réutilisation des bases spectrales, de moins en moins de nouveaux couples sont nécessaires au fil des itérations de Newton-Raphson. Enfin, la figure 5 montre l'influence du degré du chaos polynômial $p$, et donc de la dimension stochastique $P$, sur le temps de calcul de l'ensemble des systèmes linéaires. On remarque que l'algorithme proposé est très peu sensible à la dimension stochastique tandis que le temps de calcul d'une approche standard (CGS) croît très fortement avec la dimension $P$.

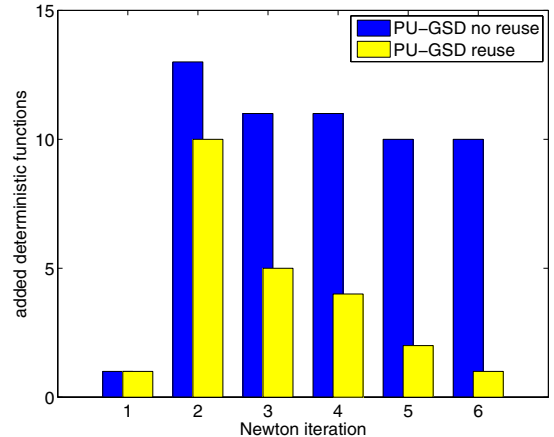

Fig. 4. Nombres de vecteurs déterministes $\boldsymbol{U}_{i}$ construits par PU-GSD au cours des itérations de Newton-Raphson : influence de la réutilisation.

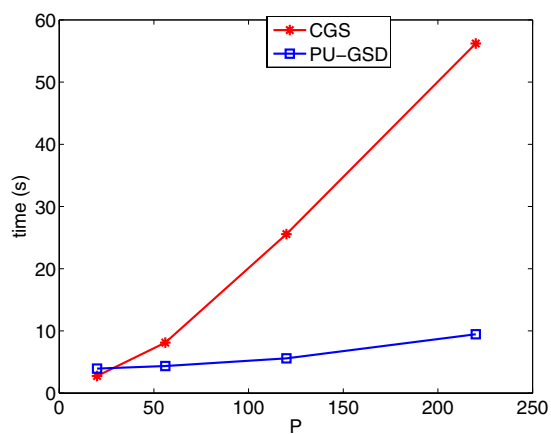

Fig. 5. Temps de calcul pour la résolution de l'ensemble des systèmes linéaires en fonction de la dimension $P$ de l'espace d'approximation stochastique.

\section{Conclusion}

Nous avons présenté une méthode de résolution de problèmes stochastiques qui consiste à générer automatiquement une base spectrale généralisée pour la décomposition de la solution. Les avantages de cette approche sont multiples. Elle réduit tout d'abord de manière drastique le coût de stockage mémoire. Elle permet aussi une réduction du temps de calcul en découplant la résolution de problèmes déterministes et de problèmes stochastiques sur bases réduites. Enfin, les bases spectrales généralisées ainsi construites peuvent être réutilisées efficacement au cours d'une résolution itérative d'un problème non-linéaire ou plus généralement dans le cadre de multi-résolutions.

Remerciements. Ces travaux sont soutenus par l'Agence National de la Recherche (ANR) dans le cadre du programme « Jeunes chercheurs $»($ projet $« \mathrm{X}-\mathrm{SFEM} »)$.

\section{Références}

[1] R. Ghanem, P. Spanos, Stochastic finite elements: a spectral approach, Springer, Berlin, 1991

[2] M. Deb, I. Babuška, J.T. Oden. Solution of stochastic partial differential equations using Galerkin finite element techniques, Computer Methods in Applied Mechanics and Engineering 190 (2001) 6359-6372 
[3] H.G. Matthies, A. Keese. Galerkin methods for linear and nonlinear elliptic stochastic partial differential equations. Computer Methods in Applied Mechanics and Engineering 194 (2005) 1295-1331

[4] R.G. Ghanem, R.M. Kruger, Numerical solution of spectral stochastic finite element systems, Computer Methods in Applied Mechanics and Engineering 129 (1996) 289-303

[5] M.F. Pellissetti, R.G. Ghanem, Iterative solution of systems of linear equations arising in the context of stochastic finite elements, Advances in Engineering Software 31 (2000) 607-616

[6] A. Keese, H.G. Mathhies, Hierarchical parallelisation for the solution of stochastic finite element equations, Computer Methods in Applied Mechanics and Engineering 83 (2005) 1033-1047

[7] A. Nouy. A generalized spectral decomposition technique to solve stochastic partial differential equations, Computer Methods in Applied Methods in Engineering, 2007, submitted

[8] A. Nouy, P. Ladevèze, Multiscale computational strategy with time and space homogenization: a radial-type approximation technique for solving micro problems, Int. J. Multiscale Computational Engineering 170 (2004) $557-574$

[9] A. Nouy, F. Schoefs, N. Moës, X-SFEM, a computational technique based on X-FEM to deal with random shapes, Eur. J. Computational Mechanics 16 (2007) 277-293

[10] I. Babuška, R. Tempone, G. E. Zouraris, Solving elliptic boundary value problems with uncertain coefficients by the finite element method: the stochastic formulation, Computer Methods in Applied Mechanics and Engineering 194 (2005) 1251-1294.

[11] C. Soize, R. Ghanem, Physical systems with random uncertainties: chaos representations with arbitrary probability measure, SIAM J. Sci. Comput. 26 (2004) 395-410

[12] O.P. Le Maître, O.M. Knio, H.N. Najm, R.G. Ghanem, Uncertainty propagation using Wiener-Haar expansions, J. Computational Physics 197 (2004) 28-57

[13] Y. Saad, Numerical methods for large eigenvalue problems, Halstead Press, New York, 1992

Retrouvez nos articles sur le site : www.edpsciences.org/meca 